\title{
Wie ungesund ist ein Ultramarathon?
}

\author{
Knechtle, Beat ; Pantelis, Nikolaidis T
}

\begin{abstract}
Wir stellen die wichtigsten Erkenntnisse zu Organschädigungen durch einen Ultramarathon zusammen. Nach einem Ultramarathon können kardiale Biomarker wie CK, CK-MB, kardiales Troponin I (cTnI) und N-terminales pro-Brain Natriuretic Peptide (NT-pro BNP) erhöht sein. Bis $80 \%$ und mehr der Finisher klagen über Verdauungsprobleme, die einer der Hauptgründe sind, einen Ultramarathon nicht zu finishen. Bis zu 90 $\%$ der Läufer, die einen Ultramarathon aufgeben, klagen über Übelkeit. Nach einem Ultramarathon steigen die Leberwerte oft an, schwerwiegende Konsequenzen bleiben meist aus. Risikofaktoren für eine Einschränkung der Nierenfunktion sind eine ausgeprägte Muskelschädigung mit Rhabdomyolyse, Dehydratation, Hypotonie, Hyperurikämie, Hyponatriämie, geringe Wettkampferfahrung sowie die Einnahme von NSARs. Ultraläufer leiden nach einem Ultramarathon oft an Infekten der oberen Atemwege. We compile the most important findings on organ damage caused by an ultra-marathon. After an ultra-marathon, cardiac biomarkers such as CK, CK-MB, cardiac troponin I (cTnI) and N-terminal pro-brain natriuretic peptides (NT-pro BNP) may be elevated. Up to $80 \%$ or more of the finishers complain about digestive problems, which are one of the main reasons to for not finishing an ultramarathon. Up to $90 \%$ of runners who give up an ultramarathon complain about nausea. After an ultramarathon, liver values often rise and usually no serious consequences remain. Risk factors for renal impairment include severe muscle damage with rhabdomyolysis, dehydration, hypotension, hyperuricemia, hyponatraemia, poor competition, and the use of NSAIDs. Ultra runners often suffer from upper respiratory infections after an ultramarathon.
\end{abstract}

DOI: https://doi.org/10.1024/1661-8157/a002943

Posted at the Zurich Open Repository and Archive, University of Zurich

ZORA URL: https://doi.org/10.5167/uzh-152261

Journal Article

Accepted Version

Originally published at:

Knechtle, Beat; Pantelis, Nikolaidis T (2018). Wie ungesund ist ein Ultramarathon? Praxis, 107(8):453-462.

DOI: https://doi.org/10.1024/1661-8157/a002943 
Knechtle $\mathrm{B}^{1,2}$, Nikolaidis PT ${ }^{3}$

2

$3 \quad{ }^{1}$ Medbase St. Gallen Am Vadianplatz, St. Gallen

$4 \quad{ }^{2}$ Institut für Hausarztmedizin, Universität Zürich, Zürich

$5 \quad{ }^{3}$ Exercise Physiology Laboratory, Nikaia, Griechenland

6

7

8

9

10 Wie ungesund ist ein Ultramarathon?

11

12

13

How unhealthy is an ultramarathon?

14

15

16

17

18

19

20

21

22

23

24

25

26

27

28

Korrespondenzadresse

29 Prof. Dr. med. Beat Knechtle

30 Facharzt FMH für Allgemeinmedizin

31 Gesundheitszentrum St. Gallen

32 Vadianstrasse 26

339001 St. Gallen

34 Switzerland

35 Telefon +41(0) 712269300

36 Telefax +41(0) 712269301

37 E-Mail beat.knechtle@hispeed.ch 


\section{Abkürzungen}

39

40 ACTH

41 ANP

42 ALAT

43 AP

44 ASAT

45 BCAA

46 BNP

47 BSR

48 CBG

49 CK

50 CK-MB

51 CRP

52 cTnl

53 DNA

54 EF

55 EKG

56 FFA

57 V-GT

58 HDL-Cholesterin

59 ITBS

$60 \quad \lg A$

61 IL-6

62 IL-8

63 IL-10

64 IL-1RA

65 IFN-y

$66 \mathrm{LDH}$

67 LDL-Cholesterin

$68 \mathrm{MCV}$

$69 \mathrm{MCH}$

$70 \mathrm{MCHC}$

71 MIBG

72 MRI

73 NSAR

74 NT-proBNP
Adrenocorticotropes Hormon

atrial natriuretic peptide

Alanin-Aminotransferase

Alkalische Phosphatase

Aspartat-Aminotransferase

Branched-chain amino acid

Brain natriuretic peptide

Blutsenkungsreaktion

Cortisol-Binding Globulin

Creatin-Kinase

Myokard Creatin-Kinase

C-reaktives Protein

kardiales Troponin I

Desoxyribonukleinsäure

ejection fraction

Elektrokardiogramm

Freie Fettsäuren (free fatty acids)

Gamma-Glutamyltransferase

High-Density-Lipoprotein Cholesterin

Iliotibiales Bandsyndrom

Immunglobulin A

Interleukin-6

Interleukin-8

Interleukin-10

Interleukin 1-Rezeptorantagonist

y-Interferon

Laktat-Dehydrogenase

Low-Density-Lipoprotein Cholesterin

Mean cell volume

Mean cell hemoglobin

Mean cell hemoglobin concentration

Meta-lodo-Benzylguanidine

Magnetic Resonance Imagining

Nicht-steroidales Antirheumatikum

$\mathrm{N}$-terminales pro-Brain Natriuretic Peptide 
75 PPI

76 ROS

77 TNF- $\alpha$

78

79

80

81

82

83

84

85

86

87

88

89

90

91

92

93

94

95

96

97

98

99

100

101

102

103

104

105

106

107

108

109

110

\section{Protonenpumpen-Inhibitor}

serum reactive oxygen species

Tumornekrosefaktor- $\alpha$ 


\section{Zusammenfassung}

113 In den letzten Jahrzehnten hat sich die Zahl der Ultraläufer vervielfacht. Wir stellen die

114 Erkenntnisse zu Schäden zusammen die ein Ultramarathon machen kann. Die häufigsten

115 Verletzungen resp. Überlastungsschäden betreffen die untere Extremität wobei

116 Sprunggelenk und Knie am häufigsten betroffen sind. Bei sehr langen Läufen kommt es zu

117 einer Anpassung mit Verdickung von Sehnen und Knorpel. Ein Ultramarathon kann zu einem

118 ausgeprägten Muskelschaden führen mit einem Anstieg von myozellulären Metaboliten wie

119 Myoglobin, LDH und CK. Dies wiederum kann zu einer Rhabdomyolyse mit Nierenversagen

120 führen. Nach einem Ultramarathon sind kardiale Biomarker wie CK, CK-MB, cTnl und NT-pro

121 BNP erhöht. Ein grosser Teil der Läufer klagt über Verdauungsprobleme und in ganz

122 seltenen Fällen kann es zu einem Leberversagen kommen.

123

124 Schlüsselwörter: Extremausdauer; Bewegungsapparat; Herz; Niere; Leber

125

126

127

128

129

130

131

132

133

134

135

136

137

138

139

140

141

142

143

144

145

146

147 


\section{Summary}

150 In recent decades, the number of ultra-runners has been multiplied. We analyze the findings

151 of previous studies on damage that be induced by an ultramarathon. Overuse injuries

152 concern the lower extremity, with the ankle and knee being the most commonly affected

153 sites. Very long runs induce thickening of tendons and cartilage as chronic adaptations. An

154 ultramarathon can lead to pronounced muscle damage with an increase in myocellular

155 metabolites such as myoglobin, LDH and CK. This in turn can lead to rhabdomyolysis with

156 kidney failure. After an ultramarathon, cardiac biomarkers such as CK, CK-MB, CTnI and NT-

157 pro BNP are elevated. A large proportion of runners complain of digestive problems and in

158 very rare cases, liver failure can occur.

159

160 Keywords: extreme endurance; musculoskeletal; heart; kidney; liver 


\section{Résumé}

186 Au cours des dernières décennies, le nombre de coureurs d'ultra-marathon s'est multiplié.

187 Nous avons rassemblé les résultats sur les dégâts que peut provoquer un ultra marathon.

188 Les blessures de surentraînement affectent le membre inférieur, la cheville et le genou étant

189 les plus souvent touchés. Les très longues courses entraînent une adaptation chronique

190 signalée par l'épaississement des tendons et du cartilage. L'ultramarathon peut provoquer

191 des dommages musculaires prononcés avec une augmentation des métabolites

192 myocellulaires tels que la myoglobine, la LDH et la CK. Cela peut à son tour conduire à une

193 rhabdomyolyse avec insuffisance rénale. Après un ultramarathon, les biomarqueurs

194 cardiaques tels que CK, CK-MB, cTnl et NT-pro BNP sont élevés. Une grande partie des

195 coureurs se plaignent de problèmes digestifs et, dans de très rares cas, une insuffisance

196 hépatique peut survenir.

198 Mots-clés: endurance extrême; musculo-squelettiques; coeur; les reins; foie 


\section{Einleitung}

223 In den letzten Jahren und Jahrzehnten erlebten Langstreckenläufer über Strecken länger als

224 ein Marathon einen richtigen Boom. Mit dem Anstieg der Anzahl Wettkämpfe sowie der Zahl

225 der erfolgreichen Finisher wurden die Ultramarathons - definiert als ein Lauf länger als die

226 Marathonstrecke von $42.195 \mathrm{~km}$ - auch für wissenschaftliche Studien interessant.

228 Ein spezielles Interesse wurde dabei an den schädlichen Einflüssen der langen Läufe auf 229 den Körper entwickelt. In dieser Übersicht stellen wir die Schädigungen nach Organen resp.

230 Organsystem zusammen und stellen uns am Schluss die Fragen wie schädlich so

231 Ultramarathons sind. Zu diesem Zweck haben wir alle wissenschaftlichen Arbeiten zu

232 Ultramarathon in Scopus (www.scopus.com) bis September 2017 gesucht. Die Stichwörter

233 waren ,ultra-marathon' sowie ,ultra marathon‘. Diese Suche führte zu über 700 Artikeln. Die

234 Arbeiten wurden dann aussortiert damit nur Fallberichte und Originalarbeiten zum Thema

235 Laufen weiter berücksichtigt wurden. Arbeiten mit Schwimmern, Radfahrern, Triathleten oder

236 anderen Sportarten wurden selektiert. Weiter haben wir nach schädigenen Einflüssen auf

237 Organe und Organysteme wie Herz, Niere, Leber und Immunsystem fokussiert. Speziell

238 wurden Arbeiten mit einer praktischen Relevanz für den Sportler, den Betreuer (Trainer) und

239 den betreuenden Arzt berücksichtigt.

\section{0}

241 Ein Ultramarathon kann zu sehr vielen pathophysiologischen Veränderungen führen. In

242 Studien an Ultraläufen zeigten sich ganz unterschiedliche Veränderungen von Laborwerten

243 wobei ein grosser Teil dieser Veränderungen auf direkte Organschäden zurückzuführen sind.

244 Bereits eine relativ kurze Laufbelastung kann zu deutlichen Veränderungen von Biomarkern

245 führen wobei die Härte resp. Intensität oder Dauer der Belastung proportional zur

246 Veränderung der Marker ist [1]. Anders ausgedrückt kann man sagen dass der Körper mit

247 einer ,Akut-Phasen-Reaktion‘ auf den Stress eines Ultramarathons antwortet [2, 3].

\section{Was passiert alles während einem Ultramarathon?}

251 Ein Ultramarathon kann zu charakteristischen Veränderungen an Biomarkern führen die auf

252 einen pathologischen Prozess im Bereich der Muskeln, der Leber, der Niere und des

253 Herzens hinweisen resp. auch von anderen Organen oder Organsystemen [1, 4, 5, 6, 7, 8, 9,

254 10] (Tabelle 1). Diese Veränderungen sind in der Regel nur vorübergehend, meist abhängig

255 von der Intensität resp. der Dauer der Belastung sowie dem Trainingszustand und

256 normalisieren sich praktisch immer im Verlauf [1]. Es gibt aber auch Biomarker die

257 unverändert bleiben trotz eines hohen physischen Stresses [11]. Wenn sich Biomarker 
während eines Ultramarathons verändern so sind diese Veränderungen nur vorübergehend und innerhalb von wenigen Tagen sind die Werte wieder im Normbereich [12] und es zeichnen sich keine längerfristigen und schädlichen Folgen ab [1, 6]. Die Veränderungen treffen sowohl jüngere wie ältere Läufer gleichermassen und sind oft unabhängig von der Lauferfahrung [7]. So führt etwa ein 48-Stunden-Lauf zu einer hypokapnischen Alkalose sowie zu einer leichten Hyperkaliämie und Hypokalzämie, aber nicht zu einer Hyponatriämie. Zudem kommt es zu einer ausgeprägten Muskelschädigung, aber zu einer Leberschädigung und keiner akuten Entzündungsreaktion. Die meisten Veränderungen der Biomarker waren 48 Stunden nach dem Lauf wieder im Normbereich [12].

Im Folgenden gehen wir nun einzelne Organe resp. Organysteme durch und schauen wie ausgeprägt diese Schädigungen sind und ob diese Schädigungen nur passager oder anhaltend sind.

\section{Schädigt ein Ultramarathon das Herz?}

Ein Ultramarathon hat einen ausgeprägt grossen Einfluss auf die Skelettmuskulatur [4] wobei vor allem die Abwärtspassagen im Lauf (exzentrische Belastung) den grössten Muskelschaden verursachen [13]. So führt etwa ein Lauf über $330 \mathrm{~km}$ mit 24‘000 Höhenmetern zu einer messbaren Entzündungsreaktion und Schwellung der Oberschenkelmuskulatur [14].

Der Skelettmuskelschaden ist gut ersichtlich an spezifischen myozellulären Metaboliten die im Blut ansteigen wie das Myoglobin [1, 7], die Laktat-Dehydrogenase (LDH) [1, 9, 15, 16, 17, 18, 19], und die Creatin-Kinase (CK) [1, 15, 17, 19, 20, 21, 22]. Aufgrund des Anstiegs der CK untersuchten mehreren Studien eine mögliche Schädigung des Herzens durch einen Ultramarathon da kardiale Biomarker wie CK, CK-MB, kardiales Troponin I (cTnl) und N-terminales pro-Brain Natriuretic Peptide (NT-pro BNP) nach einem Ultramarathon erhöht waren (Tabelle 1) [23, 24]. Zudem führt ein 90-km langer Ultramarathon zu einem CRP-Anstieg wie bei Herzinfarktpatienten [25].

Das hochsensible cTnl steigt nach einem Ultramarathon deutlich an was gleichbedeutend sein soll das ein Schaden am Herzmuskel eingetreten ist [26]. Das Ausmass des Anstiegs

292 scheint von der Laufgeschwindigkeit abhängig zu sein. Läufer die einen Ultramarathon

293 schneller finishen als langsamere Läufer weisen einen höheren Anstieg des cTnl auf [27,

294 28]. Auch die Laufgeschwindigkeit in Abhängigkeit der Länge des Ultramarathons ist von 
295 Bedeutung. So führt ein 100-km-Lauf zu deutlich höheren CK-Werten als ein Lauf über 308

$296 \mathrm{~km}[29]$.

298 Es ist bekannt dass hoch-intensive Ausdauerbelastungen zu biochemischen Veränderungen

299 führen die darauf hinweisen können dass es zu einer Muskelschädigung am Herzen

300 kommen könnte [26]. Bei Männern an einem 308 km langen Ultramarathon konnte ein

301 normaler CK-MB-Mass-Index (<5.0 ng/ml) und keine Erhöhung des cTnl nachgewiesen

302 werden und man ging davon aus dass es zur keiner Myokardschädigung gekommen war

303 trotz eines Anstiegs der CK-MB [24]. Auch bei Läufern am ,Badwater' konnte keine

304 strukturelle Schädigung des Myokards nachgewiesen werden [23]. An einem 50-Meilen und

305 100-Meilen-Lauf wurde in fast 80\% der Läufer eine erhöhte Aktivität der Serum-CK-MB

306 nachgewiesen werden wobei die Myokardszintigraphie keinen Hinweis auf eine myokardiale

307 Schädigung gab [30]. Bei Läufern vor dem Start zum, Western States Endurance Run` über

308100 Meilen waren die echokardiographischen Befunde normal [31].

310 Bei den Hormonen für die Bestimmung einer kardialen Dysfunktion kommt es zu einer

311 Veränderung von NT-pro BNP [32, 33, 34, 35] und atrial natriuretic peptide (ANP) [34]. Auch

312 die Länge des Ultramarathons ist von Bedeutung. So führen ein Lauf über $100 \mathrm{~km}$ und 308

$313 \mathrm{~km}$ zu deutlich höheren CK-Werten als ein Lauf über die klassische Marathondistanz [29].

315 Bis jetzt wird sehr kontrovers beurteilt ob eine extreme Ausdauerbelastung zu einer

316 Schädigung des Herzmuskels führt. Dass ein Ultramarathon zu einer Veränderung am

317 Herzen führt zeigte eine Studie an Läufern in einem 100-Meilen-Lauf. Im EKG kommt es für

318 das rechte Herz zu Veränderungen [36]. Die Rechtsherzableitungen zeigt für die P-Welle, die

319 ST-Strecke sowie die T-Welle eine Veränderung im EKG nach dem Lauf gegenüber dem

320 EKG vor dem Lauf [37].

322 Eine strukturelle Veränderung des Herzmuskels durch einen Ultramarathon könnte am

323 einfachsten die Echokardiographie finden. Echokardiographisch kann nach einem

324 Ultramarathon eine Einschränkung der links- [38, 39, 40] wie der rechts-ventrikulären [36, 39,

325 41, 42] Funktion nachgewiesen werden.

327 Ob einer Veränderung der link- und/oder rechtsventrikulären Funktion eintritt scheint von der

328 Länge der Belastung abhängig zu sein. Nach dem zweitägigen ,Lowe Alpine Mountain

329 Marathon' konnte eine systolische und diastolische Dysfunktion des linken Ventrikels

330 nachgewiesen werden. Humorale Marker eine Myokardschädigung waren erhöht und

331 aufgrund einer Erhöhung der Troponine ging man von einer minimalen Myokardschädigung 
aus [43]. Nach einem 24-Stunden-Lauf zeigten zwei von 20 Läufern eine leichte Erhöhung

333 der Troponine und in einem der beiden Läufer konnte echokardiographisch eine Abnahme

334 der linksventrikulären EF nachgewiesen werden [44]. Auch nach einem 89-km langen

335 Ultramarathon kam es zu einer eingeschränkten Funktion des linken sowie des rechten

336 Ventrikels [45]. Nach einem 4-Stunden-Lauf zeigte sich eine Abnahme der Aktivität des

337 MIBG (131-J Meta-lodo-Benzylguanidine) im Myokard und das Ausmass der

338 Aktivitätsabnahme korrelierte mit der zurückgelegten Strecke [46]. In einem 100-Meilen-Lauf

339 konnte kein Zusammenhang zwischen der Abnahme der linksventrikulären Funktion und der

340 Veränderung der kardialen Biomarker nachgewiesen werden [47]. Während einem 24-

341 Stunden-Lauf kam es in den letzten sechs Stunden des Rennens zu einer Abnahme der

342 linksventrikulären Funktion. Allerdings normalisierte sich die Funktion wieder innerhalb von

343 wenigen Tagen nach dem Rennen [40]. Auch in einer anderen Studie zeigte sich dass sich

344 die echokardiographischen Veränderungen nach dem Lauf innerhalb von einem Tag wieder

345 normalisierten [48]. Obwohl die Belastung eines Ultramarathons zu einer Einschränkung der

346 linksventrikulären Funktion sowie einem Anstieg von gewissen kardialen Biomarkern führt ist

347 der Mechanismus hinter diesen Veränderungen nicht bekannt [47].

349 Ein wichtiger Aspekt ist auch das Ausdauertraining zu einer Anpassung des linken Ventrikels

350 im Sinne einer linksventrikulären Hypertrophie führt [49, 50]. So konnte gezeigt werden dass

351 die Grösse des linken Ventrikels eine wichtige prädiktive Variable für die Leistung an einem

352 Ultramarathon ist [51].

353

354 Da zunehmend Frauen Ultramarathons absolvieren wurde auch untersucht ob es

355 geschlechtsspezifische Unterschiede gibt. Bei einem 100-km und einem 100-Meilen-Lauf

356 zeigte sich kein Unterschied in den echokardiographischen Veränderungen zwischen den

357 Geschlechtern [52].

Wieso kommt es zu Verdauungsproblemen?

361 Ultraläufer leiden häufig unter Verdauungsproblemen [53] und gastrointestinale Blutungen

362 nach einer Ultramarathon sind nicht ungewöhnlich [54]. Oft kommt es zu okkulten Blutungen

363 [55]. Es zeigte sich, dass Symptome des unteren Gastrointestinaltrakts mit gastrointestinalen

364 Blutungen korrelieren [54]. Allgemein kann man die Verdauungsprobleme des Ultraläufers in 365 den oberen und den unteren Gastrointestinaltrakt einteilen (Tabelle 2).

367 In einem 100-Meilen-Lauf klagten 35\% [56] und in einem Berg-Ultramarathon 43\% [53] aller 368 untersuchten Läufer über Probleme mit der Verdauung. In gewissen Läufen klagen gar $80 \%$ 
und mehr der Finisher über Verdauungsprobleme [57, 58] wobei Übelkeit am häufigsten erwähnt wird [57]. Erwiesenermassen sind Verdauungsprobleme einer der Hauptgründe warum Ultraläufer einen Ultramarathon aufgeben [59]. Bis zu 90\% der Läufer die einen

372 Ultramarathon aufgeben klagen über Übelkeit [56].

Ein möglicher Grund für diese Verdauungsprobleme könnte sein dass die Belastung während eines Ultramarathons die Motilität des Ösophagus verändert [60]. Die Endotoxinämie scheint allerdings der entscheidende Grund für Verdauungsprobleme zu sein während Faktoren wie Hyperthermie, Dehydratation und Ernährung kaum ursächlich sind [57]. Aber auch die Erfahrung scheint hier von Bedeutung zu sein. Läufer mit Verdauungsproblemen während eines Ultramarathons weisen weniger Trainingskilometer auf und machen kürzere Trainingsläufe [61]. Vom Pathomechanismus her konnte in einem 24-Stunden-Lauf gezeigt werden das Endotoxine und pro-inflammatorische Zytokine anstiegen und es auf diesen Anstieg zu einer gegenregulatorischen antientzündlichen Reaktion kam $[62,63]$.

Aus therapeutischer Sicht scheint es sehr schwierig zu sein gastrointestinale Probleme zu behandeln. Die Zufuhr von Natrium während eines 100-Meilen-Laufes hat auf jeden Fall keinen Einfluss auf Übelkeit und Erbrechen [64]. Am besten dürfte wohl ein präventives Vorgehen sein indem man an einem Ultramarathon das isst was man am liebsten hat [65]. Dabei scheint das Nahrungsmittel mit einem hohen Fettanteil sowie eine erhöhte Fettzufuhr während einem Ultramarathon deutlich weniger Verdauungsprobleme auslöst [66]. Die Einnahme eines PPI (Protonenpumpen-Inhibitor) vor einem Ultramarathon reduziert das Risiko für eine gastrointestinale Blutung während des Laufes deutlich [67].

\section{Wird die Leber geschädigt?}

Eine regelmässige körperliche Aktivität hat einen positiven Effekt auf die Leberfunktion.

397 Hingegen können sehr lange Ausdauerbelastungen - speziell unter schwierigen klimatischen 398 Bedingungen [68] - zu einem Problem für die Leber werden [69]. Speziell längere Läufe bei tiefer Intensität scheinen eher zu einem Problem für die Leber zu werden [70].

Während einem Ultramarathon kann es zu einem nachweisbaren Anstieg der Leberwerte

402 kommen [1, 15, 18, 71] (Tabelle 1). Es zeigt sich ein Anstieg der gamma-

403 Glutamyltransferase ( $\mathrm{Y}$-GT) [18, 71], der Alanin-Aminotransferase (ALAT) [1, 15, 19, 71, 72], 404 der Aspartat-Aminotransferase (ASAT) [1, 15, 19, 71, 72, 73], der alkalischen Phosphatase 405 (AP) [6, 9, 72], sowie des Bilirubins [1, 3, 6, 7, 18, 71, 74]. Diese Veränderungen sind 
abhängig von der Intensität resp. Dauer der Belastung vorübergehend und normalisieren sich im Verlauf $[1,6]$.

Es kann in sehr seltenen Fällen zu einer ausgeprägten Leberschädigung führen kann [68, 75]. Es wurde ein Fall eines jungen Läufers an einem 62-km langen Ultramarathon beschrieben bei dem es zu einem Hitzschlag kam. In der Folge kam es zu einer ausgeprägten Rhabdomyolyse und einer hypoxischen Hepatitis mit Multiorganversagen inkl. einem fulminanten Leberversagen das zu intensivmedizinischen Massnahmen führte. Im Verlauf musste eine notfallmässige Hepatektomie und orthotope Lebertransplantation durchgeführt werden [75]. In einem anderen Fall kam es bei einem 25-jährigen Mann während eines Ultramarathons zu einer Hyperthermie mit neurologischen Einschränkungen. Im weiteren Verlauf kam es zu einem akuten Leberversagen und der Patient musste intensivmedizinisch überwacht werden. Im weiteren Verlauf erholte sich der Patient wieder [68].

\section{Kommt es zu einer Nierenschädigung?}

Während Ultramarathons wird oft eine Schädigung der Niere mit Einschränkung der

424 Nierenfunktion beobachtet [76]. Aus pathophysiologischer Sicht führt der Muskelschaden zu

425 einem Einstrom von Muskelproteinen wie etwa Myoglobin in den Blutfluss. Bei gewissen

426 Umständen wie ausgeprägter Dehydratation oder Hitze kann es zu einer ausgeprägten

427 Akkumulation in der Niere mit konsekutiver Nierenschädigung kommen [77, 78, 79].

Im Bereich des Wasser- und Elektrolytstoffwechsels kommt es bei einem Ultramarathon zu ganz charakteristischen Veränderungen. Es scheint dass kurze und schnelle Ultramarathons eher zu einem Nierenproblem führen als längere Läufe mit tiefer Geschwindigkeit [70]. So

432 kommt es zu einem Anstieg von Kreatinin [70], Harnstoff [70], Harnsäure [78, 79], Natrium

433 [10, 80, 81], Kalium [7, 12, 78], Calcium [11, 12] und Phosphat [11].

435 Dann zeigt sich auch eine charakteristische Veränderung von Hormonen des Wasser- und

436 Elektrolythaushalts wie Vasopressin [33, 82, 83], Copeptin [82, 84], Oxytocin [33] und 437 Aldosteron [82] (Tabelle 1).

439 Bei Ultramarathons wird oft ein Anstieg des Kreatinins und eine vorübergehende

440 Einschränkung der Nierenfunktion beobachtet [85, 86, 87, 88, 89, 90]. In gewissen Fällen

441 erleiden alle untersuchten Läufer einer Studie eine Einschränkung der Nierenfunktion [91]. In

442 seltenen Fällen kommt es zu einer nachweislich eingeschränkten Nierenfunktion [92]. 
443 In der Regel erholt sich die Nierenfunktion innerhalb von einem [91] bis wenigen Tagen [93].

444 Bei einem Mehretappenlauf erholt sich die Nierenfunktion oft bereits wieder bis zum Start am

445 nächsten Morgen [94]. Es kann aber auch in seltenen Fällen vorkommen dass eine

446 dialysepflichtige Niereninsuffizienz entsteht [78]. Selbst wenn nun ein Läufer während einem

447 Ultramarathon eine Einschränkung der Nierenfunktion erfährt so führt ein weiterer

448 Ultramarathon nicht zwingend zu einer zusätzlichen Einschränkung der Nierenfunktion [85,

449 92].

450

451 Es zeigt sich das gewisse Personen eher ein Nierenproblem erleiden resp. ein gewisses

452 Verhalten vermehrt zu einer Nierenproblematik führen kann. Risikofaktoren für eine

453 Nierenschädigung während eines Ultramarathons sind weibliches Geschlecht, tiefes

454 Körpergewicht und deutliche Gewichtsabnahme während des Laufs [87]. Weitere

455 Risikofaktoren sind eine ausgeprägte Muskelschädigung mit Rhadomyolyse, Dehydratation,

456 Hypotonie, Hyperurikämie, Hyponatriämie, geringe Wettkampfverfahrung sowie die

457 Einnahme von NSAR [76, 77, 95].

458

459 Aus diagnostischer Sicht könnte die Teststreifen-Methode unter Feldbedingungen gute

460 Dienste leisten zum Nachweis einer Nierenschädigung durch einen Ultramarathon. In einen

461 gewissen Prozentsatz der Finisher zeigt sich eine Mikrohämaturie als Zeichen einer

462 Nierenschädigung [93]. In einer Studie mit 100-Meilen-Läufern wurden Blut- und Urinwerte

463 bei Zielankunft gemessen und verglichen. Bei den Läufern mit einer Nierenschädigung die

464 anhand der Kreatininwerte im Blut nachgewiesen wurden zeigte der Teststreifen mit einer

465 sehr hohen Spezifität und Sensitivität Eiweiss, Blut sowie ein erhöhtes spezifisches Gewicht

466 im Urin an [96].

467

468 Führt ein Ultramarathon zu einer Hämolyse?

469

470 Schon lange ist der Begriff der ,Marschhämolyse“ bekannt und dass es bei langen Läufen zu

471 einer Hämaturie kommen kann. Ein Ultramarathon kann zu einer Hämolyse führen [55] und

472 man geht davon aus dass die Hämolyse zu einem deutlichen Verlust an Erythrozyten führt

473 [97]. Nachweislich sinkt der Hämatokrit nach einem Ultramarathon [80] und das Haptoglobin

474 steigt an [55, 71]. Nicht in jedem Fall steigt das Haptoglobin nach einem Ultramarathon an,

475 möglicherweise ist die Länge des Laufes entscheidend. Zumindest kam es nach einem 60-

$476 \mathrm{~km}$ Lauf zu einer Abnahme des Haptoglobins [98].

478 An einem 166-km langen Berg-Ultramarathon konnte jedoch gezeigt werden dass es nur zu

479 einer ,Belastungsanämie‘ kommt indem die Anämie nur durch eine Expansion des 
Plasmavolumens [11] bedingt ist und nicht durch eine Reduktion des Volumens an

481 Erythrozyten [97]. Das Ausmass der Hämolyse kann aber durch entsprechendes Training

482 [99] sowie einer entsprechenden Diät mit Antioxidantien [100] deutlich reduziert werden.

484 Das Phänomen der Hämolyse bei Läufern und Ultraläufern ist kein zellulärer Schaden an den Erythrozyten. Eine Studie mit Marathon- und Ultramarathon-Läufern zeigte das Hämoglobin, Erythrozyten sowie die Erythrozyten-Indices ( $\mathrm{MCV}, \mathrm{MCH}, \mathrm{MCHC}$ ) während einem Rennen keine Veränderungen erfuhren [101]. Die vermeintliche Anämie beim Ultraläufer ist letztlich nur eine ,Verdünnungsanämie‘ [102]. Allerdings steigen die Retikulozyten nach einem 6-Tage-Lauf an [103], möglicherweise als Hinweis dass ein längerer Ultramarathon doch zu einer gewissen Schädigung der Erythrozyten führt.

Im Rahmen des Eisenstoffwechsels steigt das Ferritin an und die Transferrin-Sättigung nimmt ab [104]. Beim Ferritin konnte gezeigt werden dass der Wert bei Ultraläufern bedingt durch das Training erhöht ist und nach einem Ultramarathon bis zu zwei Wochen erhöht bleiben kann [102].

\section{Kommt es zu einer Schädigung des Immunsystems?}

Eine intensive körperliche Belastung kann teils zu ausgeprägten Gewebeschäden führen

500 [105]. So kommt es oft als Reaktion auf die Gewebeschädigung zu einer

501 Entzündungsreaktion sowie zu einer Reaktion des Immunsystems. In erfahrenen Ultraläufern kommt es nach einem Ultramarathon zu einer Veränderung der Konzentration der Immunglobuline im Blut. Diese Veränderung scheint einen Einfluss auf die Gesundheit der Ultraläufer zu haben [105].

Ein Ultramarathon scheint zu einer akuten Entzündungsreaktion zu führen mit klassischen laborchemischen Veränderungen [104, 106]. Die belastungsbedingte Entzündung beeinflusst das Knochenmark und führt zu einem gesteigerten Leukozytenturnover [107]. Es steigen Marker eines Entzündungsprozesses wie Leukozyten [1, 7, 21, 55], CRP [104, 108, 109], Ferritin [55], TNF- $\alpha$ [15, 55, 62, 110, 111], Blutsenkungsreaktion (BSR), Eisen [2], yInterferon (IFN-y) [62], der Interleukin 1-Rezeptorantagonist [111], Interleukin (IL) 1b [62, 63], IL-8 [63, 111], IL-10 [62, 63, 111] und IL-6 [11, 104, 109] an (Tabelle 1).

514 Ultraläufer leiden nach einem Ultramarathon oft an Infekten der oberen Atemwege [112,

515 113]. Es ist belegt dass Ultraläufer eher einen Infekt nach dem Wettkampf erleiden als Läufer 516 über kürzere Distanzen [114]. Die Infekte können bereits 4 Wochen vor dem Start auftreten - 
517 als Zeichen des harten Trainings - sowie noch 7-14 Tage nach Abschluss des Rennens

518 [115].

520 Von der Häufigkeit sind etwa 25-30\% der Läufer nach einem Ultramarathon betroffen [112,

521 116]. Im ,Two Oceans Marathon“ in Kapstadt, Südafrika, erlitten über 30\% der Läufer Infekte

522 der oberen Luftwege wobei eher die schnellen Läufer betroffen waren [112]. Oft haben

523 schnellere Läufer mehr Infekte als langsamere Läufer [117] während bei langsameren

524 Läufern die Häufigkeit der Infekte im Bereich von Personen einer Kontrollgruppe lag [112].

525 Von der Pathophysiologie her konnte nachgewiesen werden das ein 24-Stunden-Lauf zu

526 einer Abnahme des Speichelflusses, einer Abnahme der IgA im Speichel sowie zu einer

527 Abnahme der Sekretion von Lysozymen im Speichel führt [118, 119].

528

529 Es gibt Studien die belegen dass sowohl die Häufigkeit wie auch die Schwere eines Infektes

530 nach einem Laufe günstig beeinflusst werden können. Die Konzentration von Glutamin ist im

531 Blut nach einem Lauf um bis zu 20\% reduziert [120]. Die Einnahme von Glutamin vor und

532 nach einem Lauf senkt die Häufigkeit von Infekten [114, 120, 121].

533

534 Nebst Glutamin hat auch Vitamin C günstige Effekte auf Infekte der oberen Luftwege bei

535 Ultraläufern [122]. Die regelmässige Einnahme von Vitamin C kann die Resistenz gegenüber

536 dem gehäuften Auftreten von Infekten der oberen Luftwege verbessern und - falls trotzdem

537 ein Infekt auftritt - die Schwere des Verlaufs etwas abschwächen [113]. Selbst wenn Vitamin

538 C einen Einfluss auf einen Infekt haben kann, die Einnahme von Vitamin C führt zu keiner

539 Veränderung verschiedener Infektparameter wie Immunzellen, Interleukine, oder Interferon

540 [123]. Allerdings scheint die Höhe der zugeführten Menge an Vitamin C wichtig. Bei Läufern

541 die den ,Comrades Marathon' in Südafrika erfolgreich gefinisht haben führte zu Zufuhr von

5421 '500mg Vitamin C in der Woche vor dem Wettkampf zu einem deutlich geringeren Anstieg

543 von Interleukinen als die Zufuhr von täglich 500mg [111]. Eine höhere Menge an Vitamin C

544 lässt auch Kortisol und Adrenalin weniger stark ansteigen [124, 125].

545

546 In ganz seltenen Fällen kann ein Infekt nach einem Ultramarathon ganz desaströse

547 Ausmasse annehmen. Bei einer 51-jährigen Läuferin kam es nach einem Mehretappenlauf

548 zu einer nekrotisierenden Weichteilinfektion beider Füsse mit Septikämie. Es musste links

549 eine transmetatarsale Amputation und rechts eine Oberschenkelamputation durchgeführt

550 werden [126].

551

552

553 
554 Verdankungen

555

556 Wir danken Christian Marti, 2-facher Finisher des Trans-Europa-Laufs, für die kritische

557 Durchsicht des Manuskripts.

558

559

560

561

562

563

564

565

566

567

568

569

570

571

572

573

574

575

576

577

578

579

580

581

582

583

584

585

586

587

588

589

590

591

592

593

594

595

596

597

598

599

600

601

602

603

604

605

606 


\section{Key messages}

Durch einen Ultramarathon werden kardiale Biomarker wie CK, CK-MB, kardiales Troponin I (cTnl), atrial natriuretic peptide (ANP) und N-terminales pro-Brain Natriuretic Peptide (NT-pro BNP) erhöht

Echokardiographisch kann nach einem Ultramarathon eine Einschränkung der links- wie der rechts-ventrikulären Funktion nachgewiesen werden wobei diese Veränderung reversibel erscheint

Gastrointestinale Probleme sind sehr häufig und bis zu $90 \%$ der Läufer die einen Ultramarathon aufgeben klagen über Übelkeit

Es kann in sehr seltenen Fällen zu einer ausgeprägten Leberschädigung bis hin zum Leberversagen kommen

Bei Ultramarathons werden oft ein Anstieg des Kreatinins und eine vorübergehende Einschränkung der Nierenfunktion beobachtet wobei es sehr selten zu einer dialysepflichtigen Niereninsuffizienz kommt

Ultraläufer leiden nach einem Ultramarathon oft an Infekten der oberen Atemwege 
662

663

664

665

666

667

668

669

670

671

672

673

674

675

676

677

678

679

680

681

682

683

684

685

686

687

688

689

690

691

692

693

694

695

696

697

698

699

700

701

702

703

704

705

706

707

708

709

710

711

712

713

714

715

716

\section{Lernfragen}

Ein Ultramarathon führt

a) Immer zu einer Hyponatriämie

b) Praktisch immer im Alter zu einer Hüftarthrose

c) Zu einer Abnahme der CK im Blut

d) Zu einer Abnahme des Kreatinins im Blut

Antwort: a bis d ist falsch 
1. Bird SR, Linden M, Hawley JA. Acute changes to biomarkers as a consequence of prolonged strenuous running [Review]. Annals of Clinical Biochemistry. 2014;51(2):137-150. doi: 10.1177/0004563213492147.

2. Fallon KE. The acute phase response and exercise: The ultramarathon as prototype exercise. Clinical Journal of Sport Medicine. 2001;11(1):38-43. doi: 10.1097/00042752-200101000-00007.

3. Fallon KE, Sivyer G, Sivyer K, et al. Changes in haematological parameters and iron metabolism associated with a 1600 kilometre ultramarathon. British Journal of Sports Medicine. 1999;33(1):27-32.

4. Kim HJ, Lee YH, Kim CK. Biomarkers of muscle and cartilage damage and inflammation during a $200 \mathrm{~km}$ run [Article]. European Journal of Applied Physiology. 2007;99(4):443-447. doi: 10.1007/s00421-006-0362-y.

5. Nagel D, Seiler D, Franz H, et al. Ultra-long-distance running and the liver. International journal of sports medicine. 1990 Dec;11(6):441-5. doi: 10.1055/s2007-1024834. PubMed PMID: 2286482; Eng.

6. Wu HJ, Chen KT, Shee BW, et al. Effects of $24 \mathrm{~h}$ ultra-marathon on biochemical and hematological parameters [Article]. World Journal of Gastroenterology. 2004;10(18):2711-2714.

7. Jastrzebski Z, Zychowska M, Jastrzebska M, et al. Changes in blood morphology and chosen biochemical parameters in ultra-marathon runners during a 100-km run in relation to the age and speed of runners. International journal of occupational medicine and environmental health. 2016;29(5):801-14. doi: 10.13075/ijomeh.1896.00610. PubMed PMID: 27518889; Eng.

8. Shin KA, Kim AC, Kim YJ, et al. Effect of ultra-marathon (308 km) race on bone metabolism and cartilage damage biomarkers [Article]. Annals of Rehabilitation Medicine. 2012;36(1):80-87. doi: 10.5535/arm.2012.36.1.80.

9. Noakes TD, Carter JW. The responses of plasma biochemical parameters to a 56$\mathrm{km}$ race in novice and experienced ultra-marathon runners [Article]. European Journal of Applied Physiology and Occupational Physiology. 1982;49(2):179-186. doi: $10.1007 /$ bf02334066.

10. Burge J, Knechtle B, Knechtle P, et al. Maintained serum sodium in male ultramarathoners--the role of fluid intake, vasopressin, and aldosterone in fluid and electrolyte regulation. Hormone and metabolic research $=$ Hormon- und Stoffwechselforschung = Hormones et metabolisme. 2011 Aug;43(9):646-52. doi: 10.1055/s-0031-1284352. PubMed PMID: 21823061; Eng.

11. Fallon KE, Sivyer G, Sivyer K, et al. The biochemistry of runners in a $1600 \mathrm{~km}$ ultramarathon [Article]. British Journal of Sports Medicine. 1999;33(4):264-269.

12. Kłapcińska B, Waåkiewicz Z, Chrapusta SJ, et al. Metabolic responses to a 48-h ultra-marathon run in middle-aged male amateur runners [Article]. European Journal of Applied Physiology. 2013;113(11):2781-2793. doi: 10.1007/s00421013-2714-8.

13. Koller A, Mair J, Schobersberger W, et al. Effects of prolonged strenuous endurance exercise on plasma myosin heavy chain fragments and other muscular proteins. Cycling vs running. Journal of Sports Medicine and Physical Fitness. 1998;38(1):10-17.

14. Andonian P, Viallon M, Le Goff C, et al. Shear-wave elastography assessments of quadriceps stiffness changes prior to, during and after prolonged exercise: A 
longitudinal study during an extreme mountain ultra-marathon. PLoS ONE. 2016;11(8). doi: 10.1371/journal.pone.0161855.

15. Chiu YH, Hou SK, How CK, et al. Influence of a 100-km ultra-marathon on hepatitis B carrier runners. International journal of sports medicine. 2013;34(9):841-845. doi: 10.1055/s-0032-1331769.

16. Jastrzębski Z, Zychowska M, Jastrzębska M, et al. Changes in blood morphology and chosen biochemical parameters in ultra-marathon runners during a $100-\mathrm{km}$ run in relation to the age and speed of runners. International journal of occupational medicine and environmental health. 2015;29(5):801-814. doi: 10.13075/ijomeh.1896.00610.

17. Kanter MM, Kaminsky LA, Laham-Saeger J. Serum enzyme levels and lipid peroxidation in ultramarathon runners. Annals of Sports Medicine. 1986;3(1):3941.

18. Shin $\mathrm{K}$, Jee H, Lee $\mathrm{Y}$, et al. Effects of an extreme endurance ultra-marathon on musculoskeletal and hematologic functions. Gazzetta Medica Italiana Archivio per le Scienze Mediche. 2014;173(5):283-289.

19. Jastrzȩbski Z, Zychowska M, Radzimiński $Ł$, et al. Damage to liver and skeletal muscles in marathon runners during a $100 \mathrm{~km}$ run with regard to age and running speed. Journal of Human Kinetics. 2015;45(1):93-102. doi: 10.1515/hukin-2015-0010.

20. Carmona G, Roca E, Guerrero M, et al. Sarcomere disruptions of slow fiber resulting from mountain ultramarathon. International Journal of Sports Physiology and Performance. 2015;10(8):1041-1047. doi: 10.1123/ijspp.20140267.

21. Jee H, Park J, Oh JG, et al. Effect of a prolonged endurance marathon on vascular endothelial and inflammation markers in runners with exercise-induced hypertension. American Journal of Physical Medicine and Rehabilitation. 2013;92(6):513-522. doi: 10.1097/PHM.0b013e31829232db.

22. Suzuki M. Exercise and serum enzymes. Japanese Journal of Physical Fitness and Sports Medicine. 2002;51(5):407-422.

23. Roth HJ, Leithauser RM, Doppelmayr H, et al. Cardiospecificity of the 3rd generation cardiac troponin T assay during and after a $216 \mathrm{~km}$ ultra-endurance marathon run in Death Valley. Clinical research in cardiology : official journal of the German Cardiac Society. 2007 Jun;96(6):359-64. doi: 10.1007/s00392-0070509-9. PubMed PMID: 17453141; Eng.

24. Kim YJ, Shin YO, Lee JB, et al. The effects of running a $308 \mathrm{~km}$ ultra-marathon on cardiac markers [Article]. European Journal of Sport Science. 2014;14(SUPPL.1). doi: $10.1080 / 17461391.2011 .654267$.

25. Strachan AF, Noakes TD, Kotzenberg G, et al. C reactive protein concentrations during long distance running. British Medical Journal. 1984;289(6454):12491251.

26. Salvagno GL, Schena F, Gelati M, et al. The concentration of high-sensitivity troponin I, galectin-3 and NT-proBNP substantially increase after a 60-km ultramarathon [Article]. Clinical Chemistry and Laboratory Medicine. 2014;52(2):267-272. doi: 10.1515/cclm-2013-0601.

27. Khodaee M, Spittler J, Vanbaak K, et al. Effects of Running an Ultramarathon on Cardiac, Hematologic, and Metabolic Biomarkers. International journal of sports medicine. 2015;36(11):867-871. doi: 10.1055/s-0035-1550045. 
28. Musha H, Nagashima J, Awaya T, et al. Myocardial injury in a 100-KM ultramarathon. Current Therapeutic Research - Clinical and Experimental. 1997;58(9):587-593. doi: 10.1016/S0011-393X(97)80085-9.

29. Yoon JH, Park Y, Ahn J, et al. Changes in the markers of cardiac damage in men following long-distance and ultra-long-distance running races. Journal of Sports Medicine and Physical Fitness. 2016;56(3):295-301.

30. Matin P, Lang G, Carretta R, et al. Scintigraphic evaluation of muscle damage following extreme exercise: Concise communication [Article]. Journal of Nuclear Medicine. 1983;24(4):308-311.

31. George KP, Warburton DER, Oxborough D, et al. Upper limits of physiological cardiac adaptation in ultramarathon runners. Journal of the American College of Cardiology. 2011;57(6):754-755. doi: 10.1016/j.jacc.2010.05.070.

32. Hew-Butler T, Noakes TD, Soldin SJ, et al. Acute changes in endocrine and fluid balance markers during high-intensity, steady-state, and prolonged endurance running: Unexpected increases in oxytocin and brain natriuretic peptide during exercise. European Journal of Endocrinology. 2008;159(6):729-737. doi: 10.1530/EJE-08-0064.

33. Hew-Butler T, Jordaan E, Stuempfle KJ, et al. Osmotic and nonosmotic regulation of arginine vasopressin during prolonged endurance exercise. Journal of Clinical Endocrinology and Metabolism. 2008;93(6):2072-2078. doi: 10.1210/jc.20072336.

34. Ohba $\mathrm{H}$, Takada $\mathrm{H}$, Musha $\mathrm{H}$, et al. Effects of prolonged strenuous exercise on plasma levels of atrial natriuretic peptide and brain natriuretic peptide in healthy men. American Heart Journal. 2001;141(5):751-758. doi: 10.1067/mhj.2001.114371.

35. Tchou I, Margeli A, Tsironi M, et al. Growth-differentiation factor-15, endoglin and N-terminal pro-brain natriuretic peptide induction in athletes participating in an ultramarathon foot race. Biomarkers. 2009;14(6):418-422. doi: $10.1080 / 13547500903062976$.

36. Lord R, Somauroo J, Stembridge M, et al. The right ventricle following ultraendurance exercise: insights from novel echocardiography and 12-lead electrocardiography. European Journal of Applied Physiology. 2015;115(1):7180. doi: 10.1007/s00421-014-2995-6.

37. Lord R, George K, Somauroo J, et al. Exploratory insights from the right-sided electrocardiogram following prolonged endurance exercise. European Journal of Sport Science. 2016;16(8):1014-1022. doi: 10.1080/17461391.2016.1165292.

38. Krzemiński K, Buraczewska M, Miśkiewicz Z, et al. Effect of ultra-endurance exercise on left ventricular performance and plasma cytokines in healthy trained men. Biology of Sport. 2016;33(1):63-69. doi: 10.5604/20831862.1189767.

39. Maufrais C, Millet GP, Schuster I, et al. Progressive and biphasic cardiac responses during extreme mountain ultramarathon. American Journal of Physiology - Heart and Circulatory Physiology. 2016;310(10):H1340-H1348. doi: 10.1152/ajpheart.00037.2016.

40. Niemela KO, Palatsi IJ, Ikaheimo MJ. Evidence of impaired left ventricular performance after an uninterrupted competitive 24 hour run [Article]. Circulation. 1984;70(3 I):350-356.

41. Lord R, George K, Somauroo J, et al. Alterations in Cardiac Mechanics Following Ultra-Endurance Exercise: Insights from Left and Right Ventricular AreaDeformation Loops. Journal of the American Society of Echocardiography. 2016;29(9):879-887.e1. doi: 10.1016/j.echo.2016.05.004. 
42. Oxborough D, Shave $\mathrm{R}$, Warburton $\mathrm{D}$, et al. Dilatation and dysfunction of the right ventricle immediately after ultraendurance exercise : Exploratory insights from conventional two-dimensional and speckle tracking echocardiography. Circulation: Cardiovascular Imaging. 2011;4(3):253-263. doi: 10.1161/CIRCIMAGING.110.961938.

43. Shave RE, Dawson E, Whyte G, et al. Evidence of exercise-induced cardiac dysfunction and elevated cTnT in separate cohorts competing in an ultraendurance mountain marathon race. International journal of sports medicine. 2002 Oct;23(7):489-94. doi: 10.1055/s-2002-35069. PubMed PMID: 12402180; Eng.

44. Passaglia DG, Emed LGM, Barberato SH, et al. Acute effects of prolonged physical exercise: Evaluation after a twenty-four-hour ultramarathon [Article]. Arquivos Brasileiros de Cardiologia. 2013;100(1):21-28. doi: 10.1590/s0066782x2012005000118.

45. Chan-Dewar F, Oxborough D, Shave R, et al. Evidence of increased electromechanical delay in the left and right ventricle after prolonged exercise. European Journal of Applied Physiology. 2010;108(3):581-587. doi: 10.1007/s00421-009-1264-6.

46. Estorch M, Serra-Grima R, Carrió I, et al. Influence of prolonged exercise on myocardial distribution of 123I-MIBG in long-distance runners [Article]. Journal of Nuclear Cardiology. 1997;4(5):396-402. doi: 10.1016/s1071-3581(97)900313.

47. Scott JM, Esch BT, Shave R, et al. Cardiovascular consequences of completing a 160-km ultramarathon [Article]. Medicine and science in sports and exercise. 2009;41(1):26-34.

48. Dávila-Román VG, Guest TM, Tuteur PG, et al. Transient right but not left ventricular dysfunction after strenuous exercise at high altitude. Journal of the American College of Cardiology. 1997;30(2):468-473. doi: 10.1016/S07351097(97)00179-4.

49. Nagashima J, Musha H, Takada H, et al. New Upper Limit of Physiologic Cardiac Hypertrophy in Japanese Participants in the 100-km Ultramarathon. Journal of the American College of Cardiology. 2003;42(9):1617-1623. doi: 10.1016/j.jacc.2003.06.005.

50. Szauder I, Kovács A, Pavlik G. Comparison of left ventricular mechanics in runners versus bodybuilders using speckle tracking echocardiography. Cardiovascular Ultrasound. 2015;13(1). doi: 10.1186/s12947-015-0002-y.

51. Nagashima J, Musha $H$, Takada $H$, et al. Left ventricular chamber size predicts the race time of Japanese participants in a $100 \mathrm{~km}$ ultramarathon. British Journal of Sports Medicine. 2006;40(4):331-333. doi: 10.1136/bjsm.2005.022673.

52. Cote AT, Phillips AA, Foulds HJ, et al. Sex differences in cardiac function after prolonged strenuous exercise. Clinical Journal of Sport Medicine. 2015;25(3):276-283. doi: 10.1097/JSM.0000000000000130.

53. Rehrer NJ, Brouns F, Beckers EJ, et al. Physiological changes and gastro-intestinal symptoms as a result of ultra-endurance running. Eur J Appl Physiol Occup Physiol. 1992;64(1):1-8. PubMed PMID: 1735404; Eng.

54. Baska RS, Moses FM, Graeber G, et al. Gastrointestinal bleeding during an ultramarathon [Article]. Digestive Diseases and Sciences. 1990;35(2):276-279. doi: $10.1007 / \mathrm{bf01536777.}$ 
55. Chiu YH, Lai JI, Wang SH, et al. Early changes of the anemia phenomenon in male 100-km ultramarathoners. Journal of the Chinese Medical Association. 2015;78(2):108-113. doi: 10.1016/j.jcma.2014.09.004.

56. Stuempfle KJ, Hoffman MD. Gastrointestinal distress is common during a 161-km ultramarathon. Journal of Sports Sciences. 2015;33(17):1814-1821. doi: 10.1080/02640414.2015.1012104.

57. Stuempfle KJ, Valentino T, Hew-Butler T, et al. Nausea is associated with endotoxemia during a 161-km ultramarathon. Journal of Sports Sciences. 2016;34(17):1662-1668. doi: 10.1080/02640414.2015.1130238.

58. Wardenaar FC, Dijkhuizen R, Ceelen IJM, et al. Nutrient intake by ultramarathon runners: Can they meet recommendations? International Journal of Sport Nutrition and Exercise Metabolism. 2015;25(4):375-386. doi: 10.1123/ijsnem.2014-0199.

59. Hoffman MD, Fogard K. Factors related to successful completion of a $161-\mathrm{km}$ ultramarathon. International Journal of Sports Physiology and Performance. 2011;6(1):25-37.

60. Simons SM, Kennedy RG. Gastrointestinal problems in runners [Review]. Current sports medicine reports. 2004;3(2):112-116.

61. Glace B, Murphy C, McHugh M. Food and fluid intake and disturbances in gastrointestinal and mental function during an ultramarathon [Article]. International Journal of Sport Nutrition and Exercise Metabolism. 2002;12(4):414-427.

62. Gill SK, Ana T, Rama L, et al. Circulatory endotoxin concentration and cytokine profile in response to exertional-heat stress during a multi-stage ultra-marathon competition. Exercise Immunology Review. 2015;21:114-128.

63. Gill SK, Hankey J, Wright A, et al. The Impact of a 24-h Ultra-Marathon on Circulatory Endotoxin and Cytokine Profile. International journal of sports medicine. 2015;36(8):688-695. doi: 10.1055/s-0034-1398535.

64. Hoffman MD, Stuempfle KJ. Does oral buffered sodium supplementation reduce nausea and vomiting during an ultramarathon? Research in Sports Medicine. 2016;24(1):94-103. doi: 10.1080/15438627.2015.1126278.

65. Moran ST, Dziedzic CE, Cox GR. Feeding strategies of a female athlete during an ultraendurance running event. Int J Sport Nutr Exerc Metab. 2011 Aug;21(4):34751. PubMed PMID: 21813918; eng.

66. Stuempfle KJ, Hoffman MD, Hew-Butler T. Association of gastrointestinal distress in ultramarathoners with race diet. International Journal of Sport Nutrition and Exercise Metabolism. 2013;23(2):103-109.

67. Thalmann M, Sodeck GH, Kavouras S, et al. Proton pump inhibition prevents gastrointestinal bleeding in ultramarathon runners: A randomised, double blinded, placebo controlled study. British Journal of Sports Medicine. 2006;40(4):359-362. doi: 10.1136/bjsm.2005.024463.

68. Carvalho AS, Rodeia SC, Silvestre J, et al. Exertional heat stroke and acute liver failure: a late dysfunction. BMJ case reports. 2016 Mar 11;2016. doi: 10.1136/bcr-2016-214434. PubMed PMID: 26969359; eng.

69. Shephard RJ, Johnson N. Effects of physical activity upon the liver. European Journal of Applied Physiology. 2015;115(1):1-46. doi: 10.1007/s00421-0143031-6.

70. Shin KA, Park KD, Ahn J, et al. Comparison of Changes in Biochemical Markers for Skeletal Muscles, Hepatic Metabolism, and Renal Function after Three Types of 
Long-distance Running. Medicine (United States). 2016;95(20). doi: 10.1097/MD.0000000000003657.

71. De Paz JA, Villa JG, Lopez P, et al. Effects of long-distance running on serum bilirubin. Medicine and Science in Sports and Exercise. 1995;27(12):1590-1594.

72. Kupchak BR, Kraemer WJ, Hoffman MD, et al. The impact of an ultramarathon on hormonal and biochemical parameters in men. Wilderness \& environmental medicine. 2014;25(3):278-288. doi: 10.1016/j.wem.2014.03.013.

73. Jastrzębski Z, Zychowska M, Konieczna A, et al. Changes in the acid-base balance and lactate concentration in the blood in amateur ultramarathon runners during a 100-km run. Biology of Sport. 2015;32(3):261-265. doi: 10.5604/20831862.1163372.

74. Chou SL, Chou MY, Wang YH, et al. The impact of chronic carrier of hepatitis B virus on liver function in a 7-day ultramarathon race. Journal of the Chinese Medical Association. 2016;79(4):179-184. doi: 10.1016/j.jcma.2015.10.006.

75. Heneghan HM, Nazirawan F, Dorcaratto D, et al. Extreme heatstroke causing fulminant hepatic failure requiring liver transplantation: A case report. Transplantation Proceedings. 2014;46(7):2430-2432. doi: 10.1016/j.transproceed.2013.12.055.

76. Boulter J, Noakes TD, Hew-Butler T. Acute renal failure in four Comrades Marathon runners ingesting the same electrolyte supplement: coincidence or causation? South African medical journal = Suid-Afrikaanse tydskrif vir geneeskunde. 2011 Nov 28;101(12):876-8. PubMed PMID: 22273028; eng.

77. Clarkson PM. Exertional rhabdomyolysis and acute renal failure in marathon runners. Sports medicine (Auckland, NZ). 2007;37(4-5):361-3. PubMed PMID: 17465608; eng.

78. Uberoi HS, Dugal JS, Kasthuri AS, et al. Acute renal failure in severe exertional rhabdomyolysis. The Journal of the Association of Physicians of India. 1991 Sep;39(9):677-9. PubMed PMID: 1814900; eng.

79. Schiff HB, MacSearraigh ET, Kallmeyer JC. Myoglobinuria, rhabdomyolysis and marathon running. The Quarterly journal of medicine. 1978 Oct;47(188):463-72. PubMed PMID: 751088; eng.

80. Cejka C, Knechtle B, Knechtle P, et al. An increased fluid intake leads to feet swelling in 100-km ultra-marathoners - an observational field study [Article]. Journal of the International Society of Sports Nutrition. 2012;9. doi: 10.1186/1550-2783-9-11.

81. McKechnie JK, Leary WP, Noakes TD. Metabolic responses to a $90 \mathrm{~km}$ running race. South African Medical Journal. 1982;61(13):482-484.

82. Hew-Butler T, Hoffman MD, Stuempfle KJ, et al. Changes in copeptin and bioactive vasopressin in runners with and without hyponatremia. Clinical Journal of Sport Medicine. 2011;21(3):211-217. doi: 10.1097/JSM.0b013e31821a62c2.

83. Rogers IR, Hook G, Stuempfle KJ, et al. An intervention study of oral versus intravenous hypertonic saline administration in ultramarathon runners with exercise-associated hyponatremia: A preliminary randomized trial. Clinical Journal of Sport Medicine. 2011;21(3):200-203. doi: 10.1097/JSM.0b013e31821a6450.

84. Lippi G, Schena F, Salvagno GL, et al. Serum Copeptin and Midregion Proadrenomedullin (MR-proADM) After an Ultramarathon. Journal of Clinical Laboratory Analysis. 2015;29(1):15-20. doi: 10.1002/jcla.21720. 
1009

1010

1011

1012

1013

1014

1015

1016

1017

1018

1019

1020

1021

1022

1023

1024

1025

1026

1027

1028

1029

1030

1031

1032

1033

1034

1035

1036

1037

1038

1039

1040

1041

1042

1043

1044

1045

1046

1047

1048

1049

1050

1051

1052

1053

1054

1055

1056

1057

85. Hoffman MD, Weiss RH. Does acute kidney injury from an ultramarathon increase the risk for greater subsequent injury? Clinical Journal of Sport Medicine. 2016;26(5):417-422. doi: 10.1097/JSM.0000000000000277.

86. Hou SK, Chiu YH, Tsai YF, et al. Clinical impact of speed variability to identify ultramarathon runners at risk for acute kidney injury. PLoS ONE. 2015;10(7). doi: 10.1371/journal.pone.0133146.

87. Lipman GS, Krabak BJ, Rundell SD, et al. Incidence and Prevalence of Acute Kidney Injury during Multistage Ultramarathons. Clinical Journal of Sport Medicine. 2016;26(4):314-319. doi: 10.1097/JSM.0000000000000253.

88. Lipman GS, Krabak BJ, Waite BL, et al. A prospective cohort study of acute kidney injury in multi-stage ultramarathon runners: The biochemistry in endurance runner study (BIERS). Research in Sports Medicine. 2014;22(2):185-192. doi: 10.1080/15438627.2014.881824.

89. Lippi G, Sanchis-Gomar F, Salvagno GL, et al. Variation of serum and urinary neutrophil gelatinase associated lipocalin (NGAL) after Strenuous physical exercise. Clinical Chemistry and Laboratory Medicine. 2012;50(9):1585-1589. doi: 10.1515/cclm-2011-0954.

90. Mrakic-Sposta S, Gussoni M, Moretti S, et al. Effects of mountain ultra-marathon running on ROS production and oxidative damage by micro-invasive analytic techniques. PLoS ONE. 2015;10(11). doi: 10.1371/journal.pone.0141780.

91. Kao WF, Hou SK, Chiu YH, et al. Effects of 100-km ultramarathon on acute kidney injury. Clinical Journal of Sport Medicine. 2015;25(1):49-54. doi: 10.1097/JSM.0000000000000116.

92. Irving RA, Noakes TD, Raine RI, et al. Transient oliguria with renal tubular dysfunction after a $90 \mathrm{~km}$ running race. Medicine and Science in Sports and Exercise. 1990;22(6):756-760.

93. Kallmeyer JC, Miller NM. Urinary changes in ultra long-distance marathon runners. Nephron. 1993;64(1):119-121.

94. Lipman GS, Krabak BJ, Waite BL, et al. A prospective cohort study of acute kidney injury in multi-stage ultramarathon runners: the Biochemistry in Endurance Runner Study (BIERS). Research in sports medicine (Print). 2014;22(2):185-92. doi: 10.1080/15438627.2014.881824. PubMed PMID: 24650338; eng.

95. Seedat YK, Aboo N, Naicker S, et al. Acute renal failure in the "Comrades Marathon" runners. Renal failure. 1989;11(4):209-12. PubMed PMID: 2485484; eng.

96. Hoffman MD, Stuempfle KJ, Fogard K, et al. Urine dipstick analysis for identification of runners susceptible to acute kidney injury following an ultramarathon. Journal of Sports Sciences. 2013;31(1):20-31. doi: 10.1080/02640414.2012.720705.

97. Robach P, Boisson RC, Vincent L, et al. Hemolysis induced by an extreme mountain ultra-marathon is not associated with a decrease in total red blood cell volume [Article]. Scandinavian Journal of Medicine and Science in Sports. 2014;24(1):18-27. doi: 10.1111/j.1600-0838.2012.01481.x.

98. Lippi G, Schena F, Salvagno GL, et al. Foot-strike haemolysis after a 60-km ultramarathon. Blood Transfusion. 2012;10(3):377-383. doi: 10.2450/2012.0167-11.

99. Casoni I, Borsetto C, Cavicchi A, et al. Reduced hemoglobin concentration and red cell hemoglobinization in Italian marathon and ultramarathon runners. International journal of sports medicine. 1985;6(3):176-179. 
100. Aaseth J, Birketvedt GS. Hemolysis and rhabdomyolysis after marathon and long distance running. Immunology, Endocrine and Metabolic Agents in Medicinal Chemistry. 2012;12(1):8-13.

101. Banfi G, Roi GS, Dolci A, et al. Behaviour of haematological parameters in athletes performing marathons and ultramarathons in altitude ('skyrunners'). Clinical and Laboratory Haematology. 2004;26(6):373-377. doi: 10.1111/j.13652257.2004.00642.x.

102. Dickson DN, Wilkinson RL, Noakes TD. Effects of ultra-marathon training and racing on hematologic parameters and serum ferritin levels in well-trained athletes. International journal of sports medicine. 1982;3(2):111-117.

103. Fallon KE, Bishop G. Changes in erythropoiesis assessed by reticulocyte parameters during ultralong distance running. Clinical Journal of Sport Medicine. 2002;12(3):172-178. doi: 10.1097/00042752-200205000-00005.

104. Kasprowicz K, Ziemann E, Ratkowski W, et al. Running a 100-km ultra-marathon induces an inflammatory response but does not raise the level of the plasma ironregulatory protein hepcidin [Article]. Journal of Sports Medicine and Physical Fitness. 2013;53(5):533-537.

105. McKune AJ, Smith LL, Semple SJ, et al. Influence of ultra-endurance exercise on immunoglobulin isotypes and subclasses [Article]. British Journal of Sports Medicine. 2005;39(9):665-670. doi: 10.1136/bjsm.2004.017194.

106. Shin YO, Lee JB. Leukocyte chemotactic cytokine and leukocyte subset responses during ultra-marathon running. Cytokine. 2013;61(2):364-369. doi: 10.1016/j.cyto.2012.11.019.

107. Spiropoulos A, Goussetis E, Margeli A, et al. Effect of inflammation induced by prolonged exercise on circulating erythroid progenitors and markers of erythropoiesis. Clinical Chemistry and Laboratory Medicine. 2010;48(2):199203. doi: 10.1515/CCLM.2010.034.

108. Kim HJ, Lee YH, Kim CK. Changes in serum cartilage oligomeric matrix protein (COMP), plasma CPK and plasma hs-CRP in relation to running distance in a marathon (42.195 km) and an ultra-marathon (200 km) race [Article]. European Journal of Applied Physiology. 2009;105(5):765-770. doi: 10.1007/s00421-0080961-x.

109. Waśkiewicz Z, Kápcińska B, Sadowska-Krȩpa E, et al. Acute metabolic responses to a 24-h ultra-marathon race in male amateur runners [Review]. European Journal of Applied Physiology. 2012;112(5):1679-1688. doi: 10.1007/s00421011-2135-5.

110. Jee H, Jin Y. Effects of prolonged endurance exercise on vascular endothelial and inflammation markers. Journal of Sports Science and Medicine. 2012;11(4):719726.

111. Nieman DC, Peters EM, Henson DA, et al. Influence of vitamin C supplementation on cytokine changes following an ultramarathon. Journal of Interferon and Cytokine Research. 2000;20(11):1029-1035. doi: 10.1089/10799900050198480.

112. Peters EM, Bateman ED. Ultramarathon running and upper respiratory tract infections. An epidemiological survey [Article]. South African Medical Journal. 1983;64(15):582-584.

113. Peters EM, Goetzsche JM, Grobbelaar B, et al. Vitamin C supplementation reduces the incidence of postrace symptoms of upper-respiratory-tract infection in ultramarathon runners [Article]. American Journal of Clinical Nutrition. 1993;57(2):170-174. 
114. Castell LM. Does glutamine have a role in reducing infections in athletes? European Journal of Applied Physiology and Occupational Physiology. 1996;73(5):488-490. doi: 10.1007/BF00334429.

115. Peters EM, Shaik J, Kleinveldt N. Upper respiratory tract infection symptoms in ultramarathon runners not related to immunoglobulin status. Clinical Journal of Sport Medicine. 2010;20(1):39-46. doi: 10.1097/JSM.0b013e3181cb4086.

116. Nieman DC, Dumke CI, Henson DA, et al. Immune and oxidative changes during and following the Western States Endurance Run. International journal of sports medicine. 2003;24(7):541-547. doi: 10.1055/s-2003-42018.

117. Peters EM, Robson PJ, Kleinveldt NC, et al. Hematological recovery in male ultramarathon runners: The effect of variations in training load and running time. Journal of Sports Medicine and Physical Fitness. 2004;44(3):315-321.

118. Gill SK, Teixeira AM, Rosado F, et al. The impact of a 24-H ultra-marathon on salivary antimicrobial protein responses. International journal of sports medicine. 2014;35(11):966-971. doi: 10.1055/s-0033-1358479.

119. Gill SK, Teixeira AM, Rama L, et al. Salivary antimicrobial protein responses during multistage ultramarathon competition conducted in hot environmental conditions. Applied Physiology, Nutrition and Metabolism. 2013;38(9):977-987. doi: 10.1139/apnm-2013-0005.

120. Castell LM, Newsholme EA. The effects of oral glutamine supplementation on athletes after prolonged, exhaustive exercise. Nutrition. 1997;13(7-8):738-742.

121. Castell LM, Newsholme EA. Glutamine and the effects of exhaustive exercise upon the immune response. Canadian Journal of Physiology and Pharmacology. 1998;76(5):524-532.

122. Peters EM. Exercise, immunology and upper respiratory tract infections. International Journal of Sports Medicine, Supplement. 1997;18(SUPPL. 1):S69S77.

123. Nieman DC, Henson DA, McAnulty SR, et al. Influence of vitamin C supplementation on oxidative and immune changes after an ultramarathon. Journal of Applied Physiology. 2002;92(5):1970-1977.

124. Peters EM, Anderson R, Nieman DC, et al. Vitamin C supplementation attenuates the increases in circulating cortisol, adrenaline and anti-inflammatory polypeptides following ultramarathon running. International journal of sports medicine. 2001;22(7):537-543. doi: 10.1055/s-2001-17610.

125. Peters EM, Anderson R, Theron AJ. Attenuation of increase in circulating cortisol and enhancement of the acute phase protein response in vitamin Csupplemented ultramarathoners. International journal of sports medicine. 2001;22(2):120-126. doi: 10.1055/s-2001-11364.

126. Huang YH, Hsieh TY, Chen IC, et al. Amputation of lower limb for necrotizing softtissue infection in an ultramarathon runner. Formosan Journal of Surgery. 2014;47(2):62-65. doi: 10.1016/j.fjs.2013.10.003. 
1158 Tabelle 1: Charakteristische Veränderung von Biomarkern und Hormonen während resp. nach einem Ultramarathon

1160

\begin{tabular}{|c|c|}
\hline Skelett- und Herzmuskel & \\
\hline Creatin-Kinase (CK) & $\uparrow \uparrow-\uparrow \uparrow$ \\
\hline CK-MB & $\uparrow$ \\
\hline Myoglobin & $\uparrow$ \\
\hline Kardiale Troponine & $\uparrow$ \\
\hline Laktatdehydrogenase (LDH) & $\uparrow$ \\
\hline Leber & \\
\hline Alkalische Phosphatase (AP) & $\bar{\uparrow}$ \\
\hline Gamma-Glutamyltransferase ( $\mathrm{\gamma}-\mathrm{GT}$ ) & $\uparrow$ \\
\hline Alanin-Aminotransferase (ALAT) & $\uparrow$ \\
\hline Aspartat-Aminotransferase (ASAT) & $\uparrow$ \\
\hline Bilirubin & $\uparrow$ \\
\hline Niere, Elektrolyte, Eiweiss & \\
\hline Kreatinin & $\bar{\uparrow}$ \\
\hline Natrium & $\downarrow=$ \\
\hline Kalium & $\downarrow$ \\
\hline Kalzium & $\uparrow=$ \\
\hline Phosphat & $\uparrow$ \\
\hline Gesamteiweiss & $\downarrow$ \\
\hline Albumin & $\downarrow$ \\
\hline Blut und Blutzellen & \\
\hline Plasmavolumen & $\uparrow \downarrow$ \\
\hline Erythrozyten & $\downarrow$ \\
\hline Leukozyten & $\uparrow$ \\
\hline Haptoglobin & $\uparrow$ \\
\hline Eisen & $\uparrow$ \\
\hline Ferritin & $\uparrow$ \\
\hline Entzündung & \\
\hline C-reaktive Protein (CRP) & $\uparrow$ \\
\hline Blutsenkungsreaktion (BSR) & $\uparrow$ \\
\hline Interleukin 6 (IL-6) & $\uparrow$ \\
\hline Interleukin 8 (IL-8) & $\uparrow$ \\
\hline Interleukin 10 (IL-10) & $\uparrow$ \\
\hline Leukozyten & $\uparrow$ \\
\hline Tumornekrosefaktor (TNF- $\alpha$ ) & $\uparrow$ \\
\hline
\end{tabular}


1184 Tabelle 2: Einteilung der Verdauungsprobleme des Ultraläufers für den oberen und unteren 1185 Gastrointestinaltrakt

1186

\begin{tabular}{cc}
\hline Oberer Gastrointestinaltrakt & Unterer Gastrointestinaltrakt \\
\hline Reflux & Krämpfe im Unterbauch \\
Saures Aufstossen & Seitenstechen \\
Rülpsen & Blähungen \\
Blähungen & Stuhldrang \\
Magenschmerzen & Durchfall \\
Magenkrämpfe & Blutungen \\
Übelkeit & \\
Erbrechen & \\
\hline
\end{tabular}

1187

1188

1189

1190

1191

1192

1193

1194

1195

1196

1197

1198

1199

1200

1201
Nach Rin Cobb, Running from the Runs, www.runultra.co.uk/Articles/April-2015/Runningfrom-the-runs 